\title{
Pitfalls and Nuances of Parahisian pacing: A revisit through an interesting case.
}

\author{
Debabrata Bera ${ }^{1}$, Suchit Majumder ${ }^{2}$, Debdutta Bhattacharyya ${ }^{3}$, Rakesh Sarkar $^{4}$, and \\ Sanjeev Mukherjee ${ }^{5}$ \\ ${ }^{1}$ Bandra Holy Family Hospital and Research Centre \\ ${ }^{2}$ Apollo Gleneagles Hospital \\ ${ }^{3}$ RTIICS \\ ${ }^{4}$ Medanta The Medicity \\ ${ }^{5}$ Medica Super Speciality Hospital
}

October 1, 2020

\section{Case}

A 40-year-old gentleman underwent electrophysiology study for WPW syndrome with recurrent orthodromic atrioventricular reentrant tachycardia (AVRT). He had a right anterior accessory pathway (AP) which was ablated closer to the septum anterior to His catheter, ablation-distal (ABL) not recording any His signal during and after ablation (Appendix 1ABC). During the study, parahisian pacing (PHP) was performed before and after ablation as a routine protocol to determine the route of VA conduction (VAC). During the study 4 catheters were used: one quadripolar catheter for His region, One roving ablation catheter [for RV pacing/ mapping (MAP)/ablation], 2 decapolar catheters for coronary sinus (CS) and right atrium (RA). During PHP, both the His and MAP/ABL catheters were placed at His region. The CS catheter could not be placed fully deep inside, hence CS-12 was located near middle CS whereas the CS-34 was 2-3 cm inside proximal CS (Appendix 1BC). The decapolar catheter, placed in RA, was on the atrial aspect of tricuspid annulus, with RA-5 bipole near high right atrium (HRA). Pacing was performed at $600 \mathrm{~ms}$ cycle length (PCL) as her sinus CL was 700-750 ms. Starting at $20 \mathrm{~mA}$ current @ $2 \mathrm{~ms}$ pulse width and keeping the PCL and catheter position fixed, the current output was gradually reduced in a stepwise manner when various responses were noted. What are the responses observed in Fig.1 and Fig. 2?

\section{Commentary:}

In our case, His plus myocardium (HMc) vs. only myocardial capture (Mc) was differentiated on the basis of QRS morphology and width, although it is not the ideal way. The best way of concluding it is by analyzing whether H-signal is obscured inside or released from ventricular signal on the His catheter [1,2]. However, this was not well appreciated in our index case, hence, QRS width was used as a surrogate.

In fig 1A, the first beat represented a Mc only capture (QRS width $138 \mathrm{~ms}$ ) and second beat was a HMc (i.e. Para-His capture, QRS width $-114 \mathrm{~ms}$ ) capture. In both cases, the atrial activation sequence remained identical suggesting only one route for $V A C$. The Stimulus-A electrogram (SA) measurements were also identical. This would be called an extra-nodal response (i.e. in both the beats the VAC took place over an AP). This proves the presence of an AP, although participation of the AP in supraventricular tachycardia cannot be ascertained from PHP maneuver.

Fig 1B shows (at a different time during the same pacing protocol as Fig 1A): a very short SA (40 ms CS-12, $50 \mathrm{~ms}$ in HRA) was recorded in the first beat. The differentials of such a short SA are - (i) simultaneous 
atrial capture (SAC), (ii) Capture of ventricular end of AP. One of the ways to differentiate them is looking for 'Schmutz' after the pacing spike at the catheter near the pacing pole, MAPp and His here. But sometimes it is difficult to discriminate 'Schmutz' from myocardial V-EGM recorded at nearly the same time. However, if anytime pure his capture (PHc) without any local myocardial capture can be achieved with and without SAC (also encountered in this case, described below), the difference of EGM attached to pacing spike could be compared to delineate presence or absence of 'Schmutz', respectively. Second and easier way to validate $\mathrm{SAC}$, is to look for SA prolongation when the QRS morphology remains unchanged, as happened in the second beat (Fig 1B). Among the common responses during PHP, nodal response can also have prolongation of VA, but only during concomitant QRS widening and His-EGM release (if visible). In our case, nodal response was ruled out as the SA interval widened in spite of identical QRS width in the second beat. Hence, everything was suggestive of SAC in the first beat. Moreover, a short SA of $<60$ or $70 \mathrm{~ms}$ on the proximal CS or high right atrial electrodes respectively, is highly suggestive of SAC by itself as shown in an earlier study [3]. An 'intermittent' direct AP capture, although very rare, cannot be ruled out from above observations.

Subsequently, Fig $2 \mathrm{~A}$ shows there is SAC in both the beats. It also reveals a pure his capture (PHc) with loss of myocardial capture in the narrower second beat, as compared to HMc in the previous wider beat. The way to recognize PHc is the 12-lead ECG morphology having isoelectric segment after pacing spike. In addition, the convincing proof of $\mathrm{PHc}$ came from intracardiacs having late V-EGM recorded separately from the pacing artifact in the second beat (best appreciated in MAPd). Unless this is promptly recognized there could be a mistaken consideration of the wider beat as Mc and narrower beat as HMc, hence the interpretation for the VAC might completely change[2]. Moreover, as the ventricular EGM of the second beat is delayed and recorded separately from the pacing spike, it rendered an opportunity to analyze the 'Schmutz/SAC' $(*)$ properly (MAPp). The absence of Schmutz was distinctly noted when the SAC disappeared during PHc (Fig 2B, next discussion) and genuine VA conduction finally took place.

The next intriguing observation noted in Fig 2B during the PHP was prolongation of SA during PHc (second beat) as compared to HMc (first beat). This resulted in 48-50 ms increment in SA. In contrast the VA interval was fixed $(78 \mathrm{~ms})$ during both the beats with similar activation pattern. From this it can be inferred that the VAC was dependent on V capture and not His capture (as here the SA i.e. the HA in these two beats were not the same). Therefore, the route of VAC could be concluded to be an extra-nodal route even from these 2 beats.

An additional interesting observation was the underlying RBBB getting unmasked during PHP, when antegrade AP conduction disappeared. We believe, the prolongation of SA by $48-50 \mathrm{~ms}$ in second beat was exaggerated due to the existing/underlying RBBB as during PHc the impulse has to travel down via left bundle (LB) and exit into myocardium followed by trans-septal conduction and then VAC via right sided AP. We did not get an opportunity to demonstrate the SA prolongation in absence of RBBB as it was a fixed RBBB. We speculate, even in absence of RBBB, the SA still would have prolonged, but by a lesser magnitude, when myocardial capture is lost (Appendix 2). Hence, the PHc although infrequently encountered, can indeed help to delineate the route of VAC, irrespective of distal antegrade RBBB.

To summarize, this single case illustrates several pitfalls and challenges encountered during PHP maneuver. We speculate, many of these happened because the pacing bipole (His-D) was more proximate to the atrial side of proximal His region (Fig 3A). To complete the case, the VAC after ablation (in Fig 3B) revealed nodal response (although the PCL was $500 \mathrm{~ms}$, as sinus rate interval was approx. 600-650 ms). We believe, unwarranted findings like SAC might be avoided by placing the pacing catheter at more preferable distal His region $[1,2]$. PHc, a rare finding, can confound the interpretations if not recognized by subtle differences from HMc. However, when identified correctly, PHc can offer important electrophysiological inputs and supporting evidence regarding the route of VAC.

\section{References}

1. Hirao, K., Otomo, K., Wang, X., et al. (1996). Para-Hisian pacing. A new method for differentiating 
retrograde conduction over an accessory AV pathway from conduction over the AV node. Circulation, 94, 1027-1035

2. Sheldon SH, Li H-K, Asirvatham SJ, McLeod CJ. Parahisian pacing: technique, utility, and pitfalls. J Interv Card Electrophysiol. 2014 Aug;40(2):105-16.

3. Obeyesekere, M., Leong-Sit, P., Skanes, A., et al. (2011). Determination of inadvertent atrial capture during para-Hisian pacing. Circulation. Arrhythmia and Electrophysiology, 4, 510-514.

\section{Figure legend:}

Fig 1. Electrodes shown from top to bottom are four surface ECGs (1, aVF, V4, V1), CS12 (Middle CS) to CS34 (proximal), His distal and proximal, roving mapping catheter (MAP) right atrium RA 5(proximal, near high RA) to RA 1 (distal).

A: First beat is wider Mc beat and second beat is narrower HMc beat. The atrial activation sequence and SA measurements are similar in both beats suggestive of extra-nodal VAC.

B: Both beats are Mc but the first beat shows SAC which got released in the next beat having true VAC.

Fig 2. Electrodes shown from top to bottom are four surface ECGs (1, aVF, V4, V1), CS12 (Middle CS) to CS34 (proximal), His distal and proximal, roving mapping catheter (MAP) right atrium RA 5(proximal, near high RA) to RA 1(distal).

A: First beat is HMc with SAC. Second beat is PHc with SAC. Schmutz/SAC (marked as *) was noticeable along with the delayed separate ventricular EGM in the second beat (best seen in MAPp).

B: First beat is HMc with shorter SA. Second beat is PHc with longer SA. However, the VA i.e HA remains same in either of the beats. The absence of Schmutz is distinctly noticeable in the second beat as compared to the second beat of Fig $1 \mathrm{~B}$ and Fig $2 \mathrm{~A}$. Underlying RBBB is also unmasked.

Fig 3: Electrodes shown from top to bottom are four surface ECGs (1, aVF, V4, V1), CS12 (Middle CS) to CS34 (proximal), His distal and proximal, roving mapping catheter (MAP) right atrium RA 5(proximal, near high RA) to RA 1(distal).

A: The catheter position and electrogram just before the maneuver shows a large A-EGM almost equal to V-EGM.

B: Post-RFA para-his pacing showing nodal VA conduction. The wider first beat with Mc had shorter SA than narrower second beat (HMc) with identical atrial activation sequence.

\section{Appendix/Supporting file:}

1A: 12 lead sinus rhythm ECG with pre-excitation.

1B: Fluoroscopy image of successful site in right anterior oblique 30-degree projection.

1C: Fluoroscopy image of successful ablation site in left anterior oblique 35-degree projection. Also notice the CS and RA catheter position.

2: Cartoon showing possible effect of RBBB during $\mathrm{PHc}$ and $\mathrm{MHc}$. 

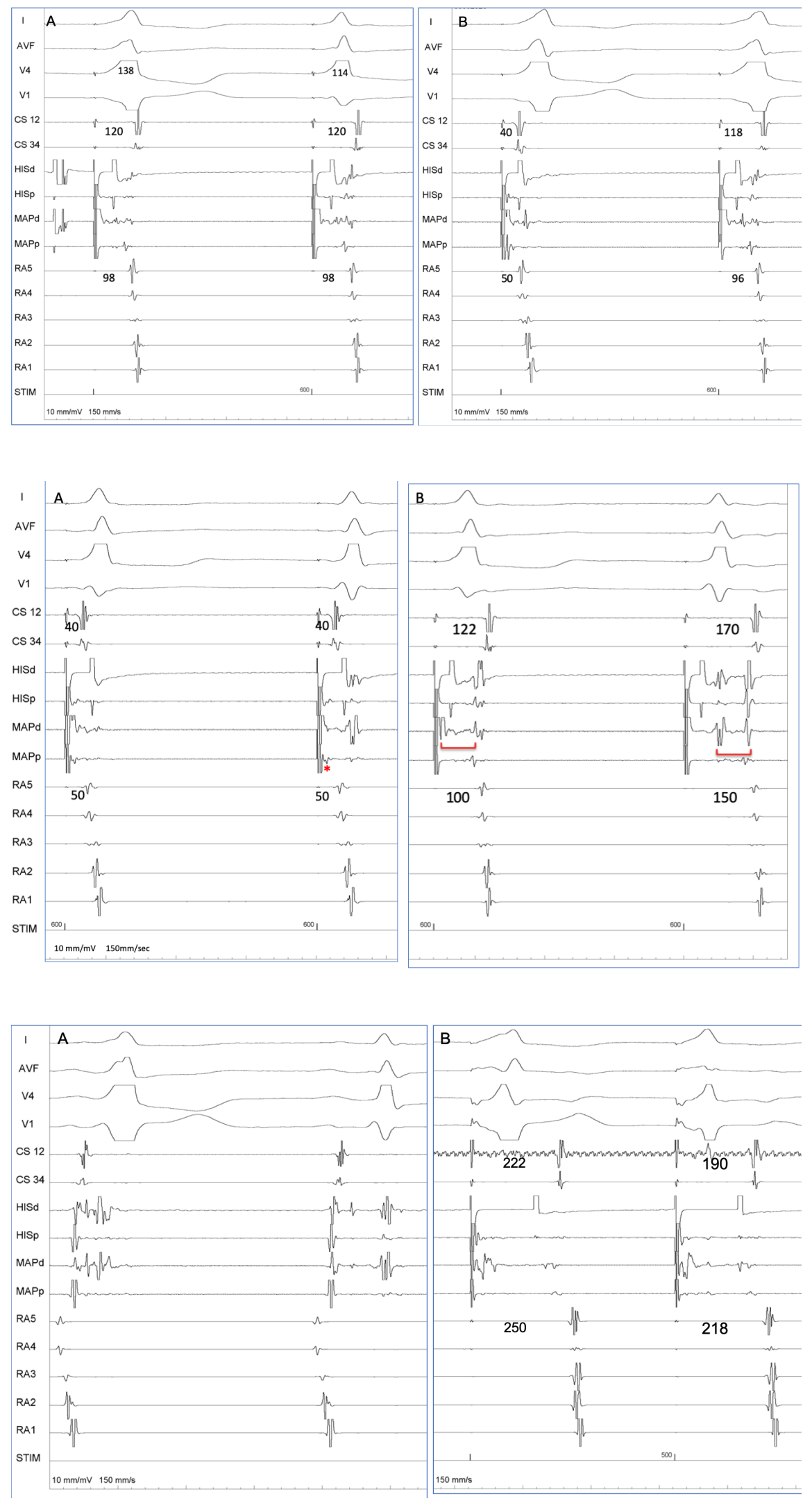